\title{
Transport and energy selection of laser generated protons for postacceleration with a compact linac
}

\author{
Stefano Sinigardi, Giorgio Turchetti, Pasquale Londrillo, and Francesco Rossi \\ Dipartimento di Fisica, Università di Bologna and INFN Sezione di Bologna, Via Irnerio, 46-I-40126 Bologna, Italy \\ Dario Giove and Carlo De Martinis \\ Dipartimento di Fisica, Università di Milano and INFN Sezione di Milano, Via Cervi, 201-I-20090 Segrate (MI), Italy \\ Marco Sumini \\ Dipartimento di Ingegneria Industriale, Università di Bologna, Viale del Risorgimento, 2-I-40136 Bologna, Italy, \\ and INFN Sezione di Bologna, Via Irnerio, 46-I-40126 Bologna, Italy
}

(Received 23 May 2012; published 1 March 2013)

\begin{abstract}
Laser accelerated proton beams have a considerable potential for various applications including oncological therapy. However, the most consolidated target normal sheath acceleration regime based on irradiation of solid targets provides an exponential energy spectrum with a significant divergence. The low count number at the cutoff energy seriously limits at present its possible use. One realistic scenario for the near future is offered by hybrid schemes. The use of transport lines for collimation and energy selection has been considered. We present here a scheme based on a high field pulsed solenoid and collimators which allows one to select a beam suitable for injection at $30 \mathrm{MeV}$ into a compact linac in order to double its energy while preserving a significant intensity. The results are based on a fully 3D simulation starting from laser acceleration.
\end{abstract}

DOI: 10.1103/PhysRevSTAB.16.031301

PACS numbers: $29.20 .-\mathrm{c}, 52.38 . \mathrm{Kd}, 41.85 .-\mathrm{p}, 84.32 . \mathrm{Hh}$

\section{INTRODUCTION}

The interaction between ultrashort electromagnetic pulses and thin foil targets has been used as an efficient way to accelerate protons and ions. With ultrashort pulses (25-50 fs) of energy less than $10 \mathrm{~J}$ and tight focusing, intensities in the range $10^{20}-10^{21} \mathrm{~W} / \mathrm{cm}^{2}$ are reached and protons up to $15-25 \mathrm{MeV}$ have been accelerated [1-3]. In a recent experiment, a maximum energy of $40 \mathrm{MeV}$ has been measured for protons [4]. In order to accelerate up to $60 \mathrm{MeV}$ (the threshold of medical interest) a proton bunch with acceptable intensity, the use of hybrid schemes based on the postacceleration of a lower energy slice has been proposed. Linacs with injection energy of $10 \mathrm{MeV}[5,6]$ and higher injection energy at $30 \mathrm{MeV}[7,8]$ have been considered. Since the size and cost of the accelerating devices decrease when the injection energy rises, the choice of injection energy at $30 \mathrm{MeV}$ appears to be a good compromise and the availability of a compact high frequency linac [9] might allow future experimental tests. Injection experiments into a radio frequency (rf) cavity of laser accelerated proton beams have been performed at very low energy [10-12] and high repetition rates. Transport lines for collimation and energy selection based

Published by the American Physical Society under the terms of the Creative Commons Attribution 3.0 License. Further distribution of this work must maintain attribution to the author(s) and the published article's title, journal citation, and DOI. on multiplets of quadrupoles [13] and high field pulsed solenoids [14-16] have been designed and experimentally checked. In the present paper we propose a start-toend simulation of the laser acceleration, transport, and postacceleration.

Two possible regimes for laser driven proton acceleration of interest for the medium size laser systems are target normal sheath acceleration (TNSA) and magnetic vortex acceleration (MVA) [17]. The first one is experimentally well established and is based on solid thin targets. The second one is based on thick targets with electron density $n \simeq n_{c}$, where $n_{c}=1.110^{21} / \lambda^{2}(\mu \mathrm{m})$ is the critical density, and has also been experimentally investigated [18].

For the TNSA regime, the conversion efficiency strongly depends on the choice of parameters of the laser-target system. Experimental data and numerical simulations show that optimal conversion efficiency is achieved by decreasing the thickness of solid targets below one micron. The energy transfer to protons in nanometric targets is improved and the maximum energy can be even doubled with respect to thick targets [19-22]. Other target designs have been proposed to avoid the use of ultrathin foils, which require a contrast greater than $10^{11}$ for intensities larger than $10^{20} \mathrm{~W} / \mathrm{cm}^{2}$. In particular, composite targets, consisting of a metal foil with a layer of quasicritical density (foam) deposited on the illuminated side, appear to improve the energy transfer from the laser to the plasma electrons and finally to the protons by the TNSA mechanism [23]. When the foam is present, we can relax the 
requirement of very high contrast and very thin foils. Numerical simulations show that the maximum proton energy in this regime reaches values comparable or even greater with respect to the TNSA regime with a bare foil [24]. The use of mass limited and curved targets to reduce the spread in energy and angle has also been proposed [25].

The MVA regime requires targets at critical or slightly overcritical density. On the experimental side, hydrogen gas jets are approaching the required parameters, and hydrogen rich aerogels might also be suitable. In this density range the medium is relativistically transparent, the laser pulse drills a channel where the electrons acceleration is mainly due to the ponderomotive force. At the exit of the target, the proton acceleration is due to an inductive electric force, caused by the expansion of a magnetic vortex created during the electron acceleration stage, and to the electron cloud moving forward as in TNSA $[17,18,26]$. Numerical simulations show that the intensity of the proton beam is comparable and the maximum energy is even higher with respect to TNSA with targets of optimal thickness. In both TNSA and MVA regimes, the energy spectrum is exponential with a cutoff. In the TNSA regime the average energy ranges between one-seventh and one-eighth of the cutoff energy. The presence of a cutoff is not an artifact due to insufficient statistics in the high energy tail of the spectrum [27]. A similar ratio is also observed in several experiments [3]. Theoretical arguments have been given to support the exponential nature of the spectrum and the presence of a cutoff for TNSA [28,29]. A more detailed discussion is provided in Appendix A.

Other regimes such as radiation pressure acceleration have been proposed [30-32], since the beam quality is higher, but there are not sufficiently consolidated experimental results to consider them for near term applications.

In the present work we choose as a model a composite target given by a thin foil with a layer of foam and a target with critical density. We take as input data the proton bunches obtained from 3D PIC simulations. The laser and target are chosen in order to end up with a maximum proton energy of at least $60 \mathrm{MeV}$. The injection into a linac for postacceleration requires the selection of an energy slice. We have chosen the slice around $30 \mathrm{MeV}$ in order to have an acceptable number of protons. The transport line is designed in order to have a selected beam of small size and emittance suitable for injection. Transport experiments and simulations aimed to applications and based on high field pulsed solenoids are reported [33,34].

The transport line we consider is based on a solenoid because it appears to be more efficient than a multiplet of quadrupoles. To reduce the angular spread of the initial beam to $50 \mathrm{mrad}$, we start with a collimator, which does not cause a relevant reduction of the intensity. To select the energy we place a second collimator after the solenoid, precisely on the focus of the protons having the energy
$30 \mathrm{MeV}$ of the selected slice. We observe that the same focus occurs for lower energy protons, but the energy separation is so large that the low energy peaks can be easily removed. The use of a collimator to perform energy selection has been independently proposed in [35].

After the transport and energy selection, the beam has a small size and transverse emittance so that $\sim 10^{7}$ particles are postaccelerated from 30 to $35 \mathrm{MeV}$ in the first module of the compact high field linac ACceleratore LIneare compatto per Prototerapia (ACLIP) [36,37]. Using five modules an energy slightly above $60 \mathrm{MeV}$ is reached without additional appreciable losses.

The proposed hybrid acceleration appears to be adequate to reach the threshold of interest for therapy. Compact fully optical systems capable of fulfilling the requirements for clinical applications are likely to be the final solution, but it is hard to estimate the time required to develop them. Therefore, hybrid solutions can represent a useful intermediate step.

\section{LASER ACCELERATION}

Experimental and computational results on proton acceleration in the TNSA regime show that the efficiency in laser absorption by a thin solid target strongly depends on the target thickness. For the typical electron density $n_{e}=400 n_{c}$ of metal foils, a very small thickness $\ell<100 \mathrm{~nm}$ can enhance the laser energy absorbed by electrons up to a factor 2 or more, compared to standard values of a few $\mu \mathrm{m}$. In a systematic $2 \mathrm{D}$ investigation of the TNSA regime [19], an empirical relation has been proposed to obtain the optimal target thickness dependence on the laser dimensionless parameter $a=e A /\left(m_{e} c^{2}\right)=$ $\alpha 0.8510^{-9} I^{1 / 2}\left(\mathrm{~W} / \mathrm{cm}^{2}\right) \lambda(\mu \mathrm{m})$, where $\alpha=1$ for linear polarization and $\alpha=1 / \sqrt{2}$ for circular polarization; the relation reads

$$
\sigma_{\mathrm{opt}}=3+0.4 a,
$$

where $\sigma=n_{e} \ell / n_{c} \lambda$ is the dimensionless areal density of the target. For large values of $a$ the constant term can be neglected and this relation suggests that the optimal thickness is close to the relativistic transparency threshold given by $\pi \sigma=a$. The same relation appears to hold also for 3D simulations [38]. Another feature of TNSA regime is the exponential nature of the energy spectrum, see Appendix A for more details:

$$
\frac{d N}{d E}=\frac{N_{0}}{E_{0}} e^{-E / E_{0}}, \quad E_{\min }<E<E_{\max } .
$$

$d N / d E=0$ if $E<E_{\min }$ or $E>E_{\max }$. If $E_{\min } \ll E_{0} \ll$ $E_{\max }$ then $N_{0}$ is the total number of protons and $E_{0}$ is the average energy. Supposing $E_{\max }=60 \mathrm{MeV}$ and $E_{0}=$ 7.5 MeV, the number of protons in a $1 \mathrm{MeV}$ slice at $30 \mathrm{MeV}$ is $e^{4} \sim 50$ times larger than in a slice at $60 \mathrm{MeV}$. 
We have performed extensive numerical investigations in $3 \mathrm{D}$ using the acceleration by laser and dynamics of charged particles (ALADYN) code [39-42] to provide quantitative estimates on the proton beams accelerated in the TNSA and MVA regimes.

As a particular example for the TNSA regime, we consider a linearly polarized laser pulse of duration $\tau=25$ fs full width half maximum (FWHM), wavelength $\lambda=0.8 \mu \mathrm{m}$ and power $P=270 \mathrm{TW}$. With a waist $w=3 \mu \mathrm{m}$ the intensity is $I=210^{21} \mathrm{~W} / \mathrm{cm}^{2}$ which corresponds to $a=30$. The target we consider is an $\mathrm{Al}$ foil fully ionized with electron density $n_{e}=40 n_{c}$ of thickness $\ell=0.5 \mu \mathrm{m}$. The layer of contaminants is hydrogen $\mathrm{H}$ of thickness $0.05 \mu \mathrm{m}$ and density $n_{e}=9 n_{c}$. The transverse size of the contaminants is chosen comparable to the size of the focal spot, to illustrate how a mass limited target reduces energy spectrum width and the angular spread. In the upper frame of Fig. 1 we show the energy spectrum $d N / d E$ of this mass limited target.
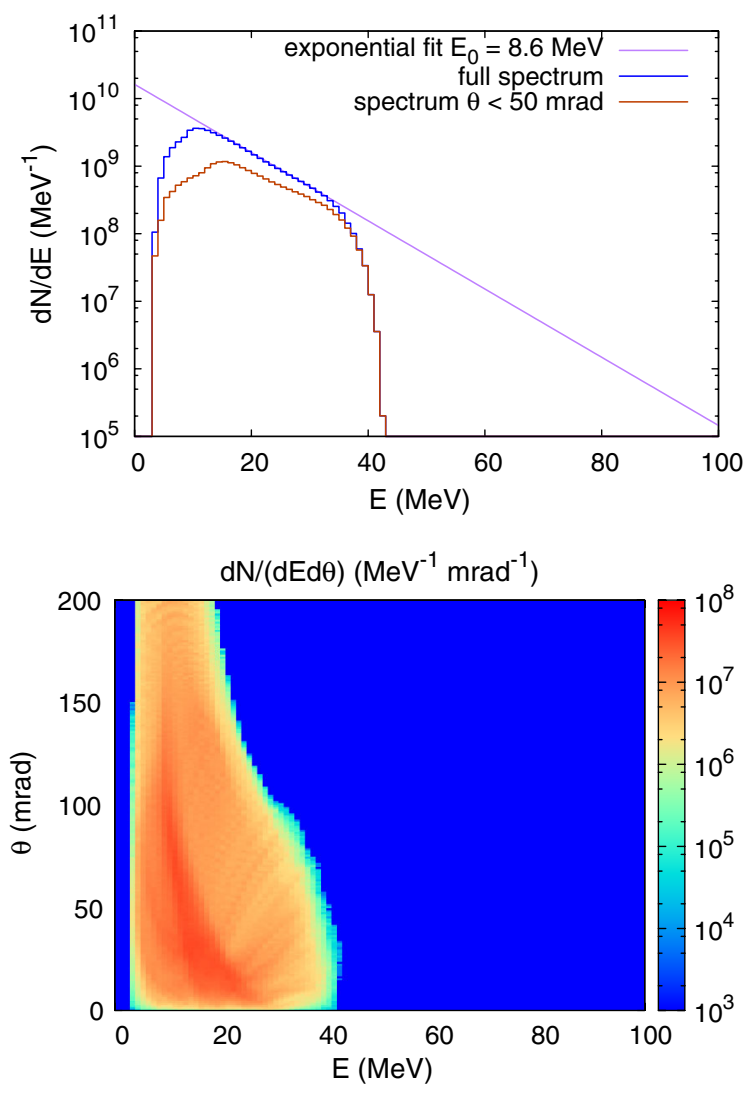

FIG. 1. Upper frame: Plot in a logarithmic scale of the proton initial energy spectrum (blue line) and after an angular selection $\theta<50 \mathrm{mrad}$ (light brown line) for the mass limited target (TNSA) with $a=30$. The values refer to the actual number of particles; the energy interval is divided into 100 bins so that each energy bin corresponds to $\Delta E=1 \mathrm{MeV}$. Lower frame: Initial energy-angle distribution of the bunch in a logarithmic color scale. The energy range is divided into 100 bins, while the angular range is divided into 200 bins, so that each energy bin corresponds to $\Delta E=1 \mathrm{MeV}$, each angle bin to $\Delta \theta=1 \mathrm{mrad}$.
The average energy is $E_{0}=8.6 \mathrm{MeV}$. In the lower frame of Fig. 1 we display the energy-angle distribution $d N / d E d \theta$, where $\theta=\arctan \left(p_{\perp} / p_{z}\right)$ having denoted by $p_{\perp}=\left(p_{x}^{2}+p_{y}^{2}\right)^{1 / 2}$ the transverse component and by $p_{z}$ the longitudinal component of the momentum of each proton macroparticle.

The optimal thickness for the electron density chosen above is $\ell=0.3 \mu \mathrm{m}$ and decreases by 1 order of magnitude for a real metal foil. In this case an extremely high contrast would be required. For this reason we have considered, as a model for transport and postacceleration, a composite target where on the illuminated side of the foil is deposited a layer of foam having quasicritical density [23]. The laser parameters are the same as for the previous model. The ionization state of the $\mathrm{Al}$ foil is limited to the ninth electron, the thickness $\ell=0.5 \mu \mathrm{m}$ and the electron density $n_{e}=40 n_{c}$. In this case a lower ionization state of the foil is justified since the absorption of laser energy occurs mainly in the foam layer. The foam layer is $\mathrm{H}$ fully ionized, $2 \mu \mathrm{m}$ thick with density $n_{e}=2 n_{c}$, and the contaminants are still modeled by a $\mathrm{H}$ layer, $0.05 \mu \mathrm{m}$ thick, of density $n_{e}=9 n_{c}$. The presence of the foam eases the constraints on the foil thickness and density so that
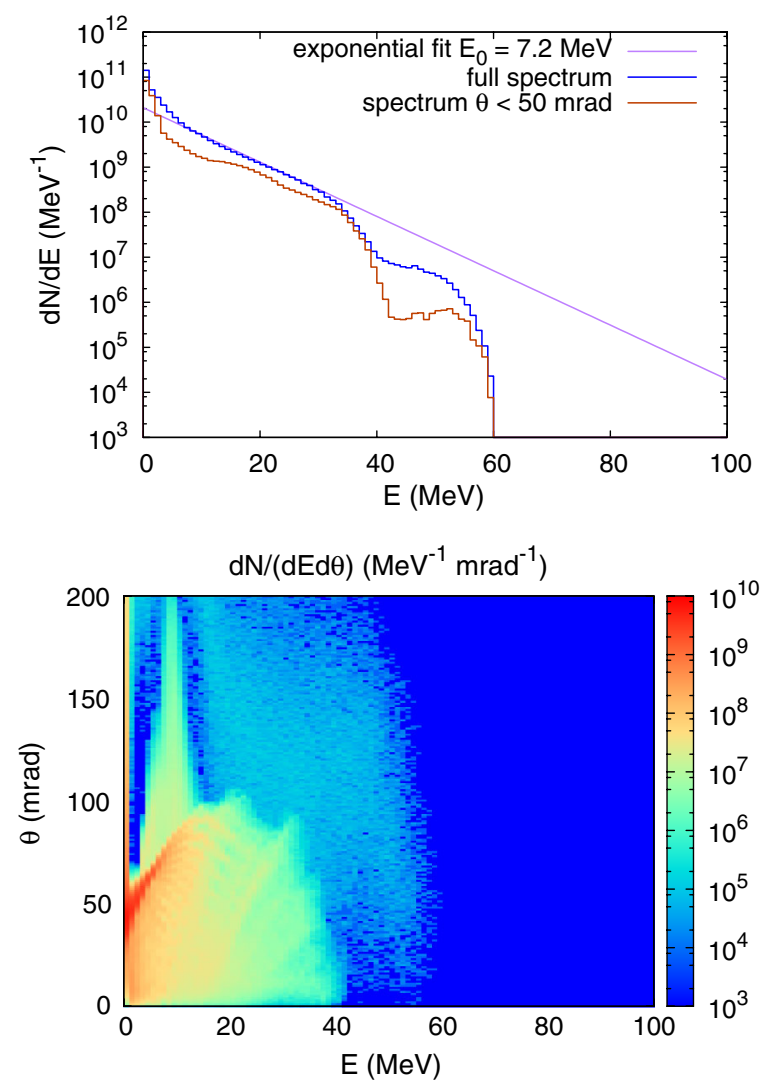

FIG. 2. Upper frame: Plot in a logarithmic scale of the proton initial energy spectrum (blue line) and after an angular selection $\theta<50 \mathrm{mrad}$ (light brown line) for the composite foam + foil target (TNSA) with $a=30$. Lower frame: Initial energy-angle distribution of the bunch in a logarithmic color scale. 
our model becomes reasonably realistic. In the upper frame of Fig. 2, we show the proton energy spectrum for the composite target, where the maximum energy is $60 \mathrm{MeV}$ and the average energy is $7.2 \mathrm{MeV}$; we also compare the full spectrum with the energy spectrum obtained with a cutoff in the angle $\theta$ at $50 \mathrm{mrad}$. In the lower frame we show the energy-angle distribution. Even though the angular spread is larger with respect to the mass limited target most of the protons have $\theta<100 \mathrm{mrad}$ except for very low energies.

The longitudinal resolution for overcritical targets has been chosen in order to resolve the skin depth in the foil, which in our case is $\ell_{\text {skin }}=\lambda /\left[2 \pi\left(n_{e} / n_{c}-1\right)^{1 / 2}\right] \simeq$ $20 \mathrm{~nm}$. The computational box is $L_{z}=22 \mu \mathrm{m}$, and $L_{x}=$ $L_{y}=32 \mu \mathrm{m}$ transversally. The grid size is $\Delta z=10 \mathrm{~nm}$, $\Delta x=\Delta y=30 \mathrm{~nm}$ so that the number of cells is $2.5 \times 10^{9}$ whereas the total number of macroparticles describing the electrons is $2 \times 10^{9}$ and the number of ions is correspondingly chosen on the base of the ionization state. Transversally the laser pulse and electron density variations are milder so that we can allow a space resolution larger than the skin depth.
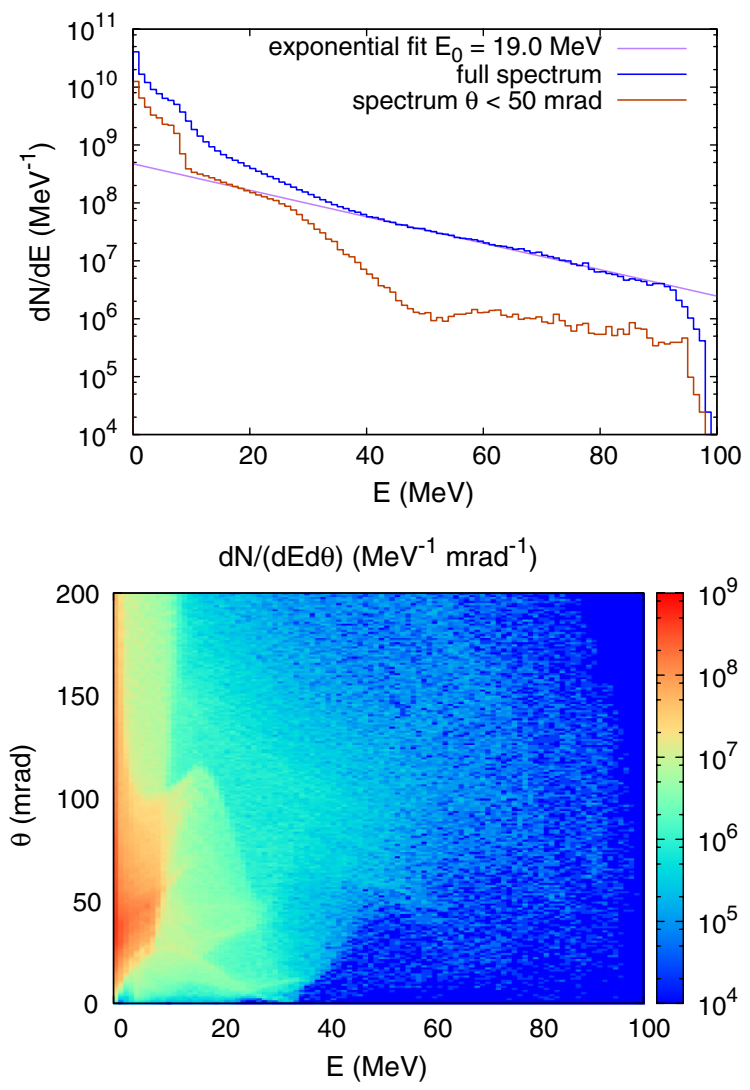

FIG. 3. Upper frame: Plot in a logarithmic scale of the proton initial energy spectrum (blue line) and after an angular selection $\theta<50 \mathrm{mrad}$ (light brown line) for the target with critical density (MVA) with $P=155 \mathrm{TW}$ and $a=19$. Lower frame: Initial energy-angle distribution of the bunch in a logarithmic color scale.
For the quasicritical targets, where the MVA regime dominates, the key laser parameter is power rather than intensity [17]. We have considered a circularly polarized laser pulse with $P=155 \mathrm{TW}$ of duration 25 fs FWHM. The waist is $2.5 \mu \mathrm{m}$ so that the intensity is $I=1.58 \times$ $10^{21} \mathrm{~W} / \mathrm{cm}^{2}$ which corresponds to $a=19$. The target thickness is $40 \mu \mathrm{m}$ and its electron density is $n=n_{c}$. In this case PIC simulations provide a maximum energy $E_{\max } \sim 100 \mathrm{MeV}$. The full energy spectrum and the spectrum with a cutoff in the angle at $50 \mathrm{mrad}$ are shown in the upper frame of Fig. 3. The distribution in the energyangle plane is presented in the lower frame of the same figure: it shows that the angular spread is higher than in the overcritical case. For this configuration the computational box is $L_{z}=40 \mu \mathrm{m}$ and $L_{x}=L_{y}=20 \mu \mathrm{m}$ with a grid size $\Delta_{z}=25 \mathrm{~nm}$ and $\Delta_{x}=\Delta_{y}=50 \mathrm{~nm}$. The total number of cells is $2.5 \times 10^{8}$ and the average number of macroparticles per cell is 8 so that the total number is $2 \times 10^{9}$ (the initial distribution is uniform).

The laser Frascati laser for acceleration and multidisciplinary experiments (FLAME), presently available in Frascati Istituto Nazionale di Fisica Nucleare (INFN) laboratories, is expected to reach intensities up to $2 \times 10^{21} \mathrm{~W} / \mathrm{cm}^{2}$. The laser induced light ions acceleration (LILIA) experiment on proton acceleration is planned to test various targets in view of a transport and postacceleration experiment.

\section{TRANSPORT AND ENERGY SELECTION}

The propagation of a proton bunch with a wide energy spectrum and a significant angular spread leads to a rapid growth of the longitudinal size and to a transverse expansion. Let $E$ denote the kinetic energy of a proton and $p=$ $\left(p_{x}^{2}+p_{y}^{2}+p_{z}^{2}\right)^{1 / 2}$ the total momentum so that $E / m c^{2}=$ $\left[1+p^{2} /(m c)^{2}\right]^{1 / 2}-1$ (at the relatively small energies involved here $E \simeq p^{2} / 2 m$ ). The angular deviations with respect to the propagation axis $z$ can be defined, letting $s=c t$, in two different ways: $x^{\prime}=d x / d s=p_{x} / p$, $y^{\prime}=d y / d s=p_{y} / p$ or $x^{\prime}=d x / d z=p_{x} / p_{z}$ and $y^{\prime}=$ $d y / d z=p_{y} / p_{z}$. In the paraxial approximation there is no difference. For the distributions obtained from PIC simulations it is found that $\left|x^{\prime}\right|,\left|y^{\prime}\right|<0.1$ (at least for energies above $1 \mathrm{MeV}$ ) using the second definition. As a consequence, $p_{z}$ and $p$ differ by less than $1 \%$, since $p_{z} / p=\left(1+x^{\prime 2}+y^{12}\right)^{-1 / 2}$, and no appreciable difference with respect to the first definition is found. The energy spread does not allow to define a one to one correspondence between time (or $s=c t$ ) and $z$. Indeed at the same time the particles are spread out along $z$ due to the large spectrum of velocities. For a monochromatic beam, the evolution on a linear transport line preserves the areas in each phase plane $\left(x, x^{\prime}\right)$ and $\left(y, y^{\prime}\right)$ in the absence of linear coupling. Elliptical distributions are rotated and stretched keeping the product of the semiaxis unchanged in order to 
preserve the area. If the energy spectrum is not monochromatic, the evolution with $z$ of the transverse phase planes distributions does not exhibit geometric invariants. This is the case only for each monochromatic energy slice.

When the full beam is considered, a given point $z$ is reached at different times by particles having different energies. The energy slice analysis of transport is cumbersome and we adopt a strategy based on the computation of the rms invariants in order to characterize its properties. At any time we compute the averages $\left\langle x_{i}\right\rangle$, variances $\sigma_{i}^{2}$ of the space variables and the emittances $\epsilon_{i}$. Letting $x_{1}=x, x_{2}=$ $y, x_{3}=z$ they are defined by

$$
\begin{gathered}
\sigma_{i}^{2}=\left\langle\left(x_{i}-\left\langle x_{i}\right\rangle\right)^{2}\right\rangle, \\
\epsilon_{i}^{2}=\left\langle\left(x_{i}-\left\langle x_{i}\right\rangle\right)^{2}\right\rangle\left\langle\left(x_{i}^{\prime}-\left\langle x_{i}^{\prime}\right\rangle\right)^{2}\right\rangle \\
+-\left[\left\langle\left(x_{i}-\left\langle x_{i}\right\rangle\right)\left(x_{i}^{\prime}-\left\langle x_{i}^{\prime}\right\rangle\right)\right\rangle\right]^{2} .
\end{gathered}
$$

These averages are computed at each time step but to display them we use $\langle z\rangle$ rather than $t$ itself. Indeed the longitudinal position of the center of mass of the beam $\langle z\rangle$ provides a more intuitive picture of the propagation of the beam. When its longitudinal size, measured by $\sigma_{z}$, becomes large the interpretation of the dependence of the position variances and emittances on $\langle z\rangle$ is no longer intuitive as for a short bunch.

The average and variance of $z$ can be easily estimated for an exponential energy spectrum. Letting $\rho(E)$ be the spectrum normalized to 1 , the mean value and variance square root of $z$ are given by $\langle z\rangle=t\langle v\rangle$ and $\sigma_{z}=t \sigma_{v}$ assuming that $v_{z} \simeq v=(2 E / m)^{1 / 2}$. The spectrum is given by $\rho(E)=E_{0}^{-1} e^{-E / E_{0}}$ if we neglect the upper cutoff and $E_{0}$ denotes the average energy. If the support of the spectrum is in the interval $\left[E_{1}-\Delta E, E_{1}+\Delta E\right]$ then, letting $\delta_{1}=\Delta E / E_{1}$ and $v_{1}=\left(2 E_{1} / m\right)^{1 / 2}$, we find that

$$
\langle z\rangle=v_{1} t\left[1+O\left(\delta_{1}^{2}\right)\right], \quad \sigma_{z}=v_{1} t \frac{\delta_{1}}{\sqrt{12}}=\langle z\rangle \frac{\delta_{1}}{\sqrt{12}}
$$

provided that $\delta_{1}$ is small. More details on the derivation of these relations are given in Appendix A. The spectrum may be filtered to reduce its support to a smaller interval, by using a suitable focusing and collimation system. Supposing that $\langle z\rangle$ is $1 \mathrm{~m}$ and that $\delta_{1}=10 \%$ then $\sigma_{z}$ is close to $3 \mathrm{~cm}$. If $\delta_{1}=1 \%$ then $\sigma_{z}=3 \mathrm{~mm}$ and the whole bunch is suitable for injection into a high frequency rf cavity. In such cases the use of $\langle z\rangle$ as independent variable is very appropriate.

The early propagation of the beam, from $t=0$ up to $t \sim 0.15 \mathrm{ps}$, where the acceleration process terminates, is described by the PIC simulation. The subsequent propagation up to a few ps is mainly ballistic, but the interaction of electrons and protons still plays a role at least for charge neutralization. The propagation of the beam is treated by neglecting the electrons, part of which is still comoving with the protons. The separation of protons becomes large due to free motion and the dilution of the proton bunch allows, at first approximation, to neglect space charge effects. It would be desirable to model these effects but the available simulation schemes are still inadequate to fully address this issue.

We analyze the transport by taking the PIC phase space distribution specified by the phase space coordinates of each numerical particle and neglect the Coulomb interaction at first. Given the specific peculiarities of the laser produced proton beam, we find that our basic requirements are completely fulfilled by high field pulsed solenoids. Cylinders of millimetric size in the proximity of the interaction region have been proposed to focus the beam with plasma lenses [43], created by shooting them with a lower intensity synchronized laser pulse. In spite of their effectiveness, the difficulty of using them with the required repetition rate suggests to not consider them in a transport line based on consolidated technologies.

\section{The solenoid}

We consider first a transport line made of a drift of length $D$ and a circular collimator of radius $r$, followed by a solenoid of length $L$. The field of a solenoid starting at $z=D$ and ending at $z=D+L$ is

$$
\mathbf{B}=-\frac{r}{2} B_{z}^{\prime}(z) \mathbf{e}_{r}+B_{z}(z) \mathbf{e}_{z},
$$

where $B_{z}$ is given by

$$
\begin{gathered}
B_{z}(z)=B_{0}[H(z-D)-H(z-D-L)], \\
H(z)=\frac{1}{1+e^{-z / \eta}} .
\end{gathered}
$$

For $\eta \rightarrow 0$, we obtain the sharp edge solenoid, whose field has a jump at the entrance and at the exit. The Larmor space frequency $\Omega_{L}$ is given by

$$
\Omega_{L}=\frac{e B_{0}}{2 m c^{2}}=0.01597 B_{0}[\text { Tesla }] \mathrm{cm}^{-1} .
$$

The analytic treatment is elementary only in the sharp edge limit. In this case, we have a focusing in both phase planes $\left(x, x^{\prime}\right)$ and $\left(y, y^{\prime}\right)$ followed by a rotation in the $(x, y)$ and $\left(x^{\prime}, y^{\prime}\right)$ planes. Letting $\beta_{z}=v_{z} / c \simeq p_{z} / m c$, we define

$$
\sqrt{k}=\frac{\Omega_{L}}{\beta_{z}}=\frac{e B_{0}}{2 m c^{2} \beta_{z}}, \quad \alpha=\sqrt{k} L .
$$

The particle trajectories at the exit of the solenoid are parallel to the $z$ axis if $k^{1 / 2} \tan \alpha=1$. At a distance $D_{1}$ from the end of the solenoid, the particle crosses the transverse plane at $\left(x_{1}, y_{1}\right)$ such that

$$
x_{1}^{2}+y_{1}^{2}=A^{2}(E)\left(x_{0}^{\prime 2}+y_{0}^{\prime 2}\right)
$$

where 


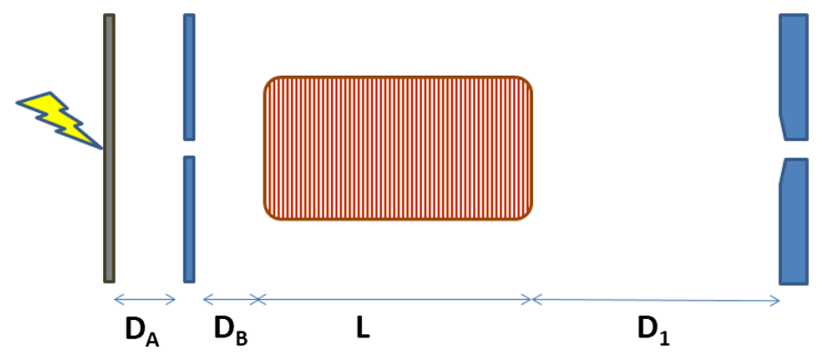

FIG. 4. Schematic drawing of the transport line: $D_{A}=D_{B}=$ $10 \mathrm{~mm}, D_{1}=510 \mathrm{~mm}, L=300 \mathrm{~mm}$, first iris radius $=0.5 \mathrm{~mm}$, second iris radius $=0.5 \mathrm{~mm}$, second iris minimum thickness $=$ $5 \mathrm{~mm}$.

$$
A=D \cos \alpha+k^{-1 / 2} \sin \alpha-D D_{1} k^{1 / 2} \sin \alpha+D_{1} \cos \alpha
$$

As a consequence, the focus for particles of a given energy occurs at a distance $D_{1}$ such that $A=0$. If we put a collimator of radius $r$ at $z=D+L+D_{1}$, only the particles having $A\left(x_{0}^{\prime 2}+y_{0}^{\prime 2}\right)^{1 / 2}<r$ will get through and energy selection is achieved. The particles distribution obtained with a sharp edge solenoid is analytically derived in Appendix B, where the presence of secondary peaks is explained. The numerical solution of the equations of motion shows that for a solenoid with fringe fields the
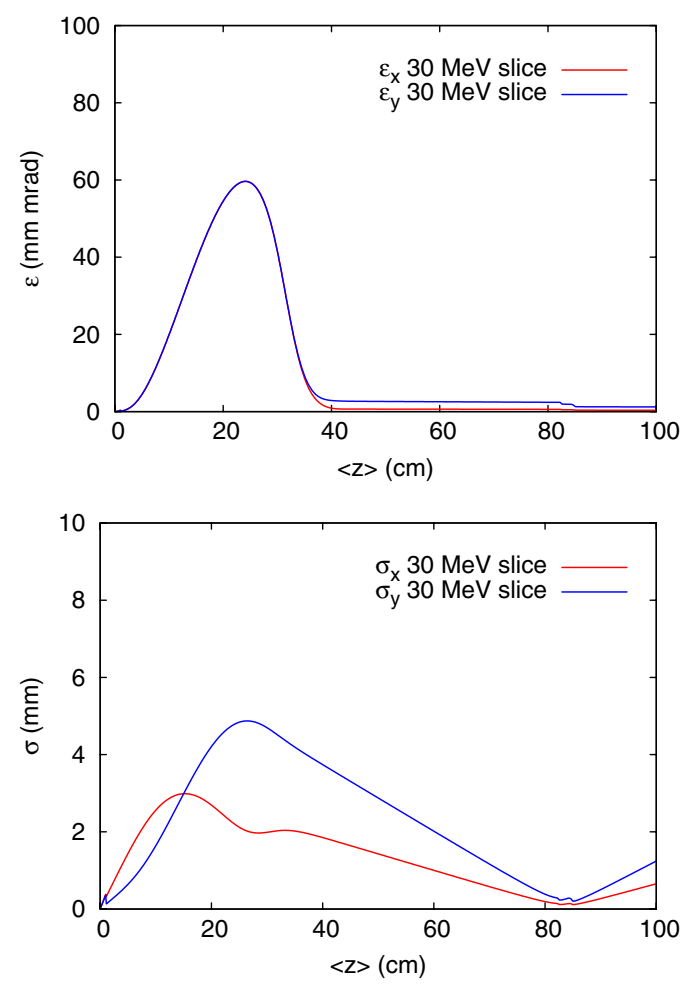

FIG. 5. Upper frame: Plot of the transverse emittances $\epsilon_{x}, \epsilon_{y}$ in $\mathrm{mm} \mathrm{mrad}$ as a function of $\langle z\rangle$ in $\mathrm{cm}$ for an energy selection of the beam $29<E<31 \mathrm{MeV}$. Lower frame: Plot of the rms beam sizes $\sigma_{x}, \sigma_{y}$ in $\mathrm{mm}$ for the same energy selection. The focus of the solenoid for this quasimonoenergetic beam is at $z=83 \mathrm{~cm}$. situation is the same with slightly modified values of the parameters. The second collimator is chosen as an Al slab $1 \mathrm{~cm}$ thick, such that all protons up to $60 \mathrm{MeV}$ are stopped. The hole is a cone with an aperture of $1 \mathrm{~mm}$ radius, small basis of $0.5 \mathrm{~mm}$ radius and height of $5 \mathrm{~mm}$, followed by a cylinder of $0.5 \mathrm{~mm}$ radius and $5 \mathrm{~mm}$ height. The thickness of $5 \mathrm{~mm}$ is sufficient to stop protons up to $35 \mathrm{MeV}$. Our transport line consists in a drift of $1 \mathrm{~cm}$, followed by a collimator of radius $r=0.5 \mathrm{~mm}$ and then another drift of $1 \mathrm{~cm}$ so that altogether the drift length is $D=$ $2 \mathrm{~cm}$. It follows a solenoid of length $L=30 \mathrm{~cm}$ with $B=10$ Tesla, having a fringe field with $\eta=2 \mathrm{~cm}$. The particles with energy $E=30 \mathrm{MeV}$ are focused at $z=83 \mathrm{~cm}$ namely after a drift of $D_{1}=51 \mathrm{~cm}$ from the end of the solenoid, and there the second collimator is located, see Fig. 4.

We first perform a numerical selection of the spectrum at $E=30 \pm 1 \mathrm{MeV}$ and follow the bunch along this transport line.

The corresponding transverse emittances and transverse size rms values are shown in Fig. 5 where we choose $\langle z\rangle$ as an independent variable. Along the same transport line, we have propagated all the protons in the energy interval $[3,60] \mathrm{MeV}$. The transverse emittances and transverse rms size values are reported in Fig. 6. We observe oscillations in the transverse rms sizes and emittances because
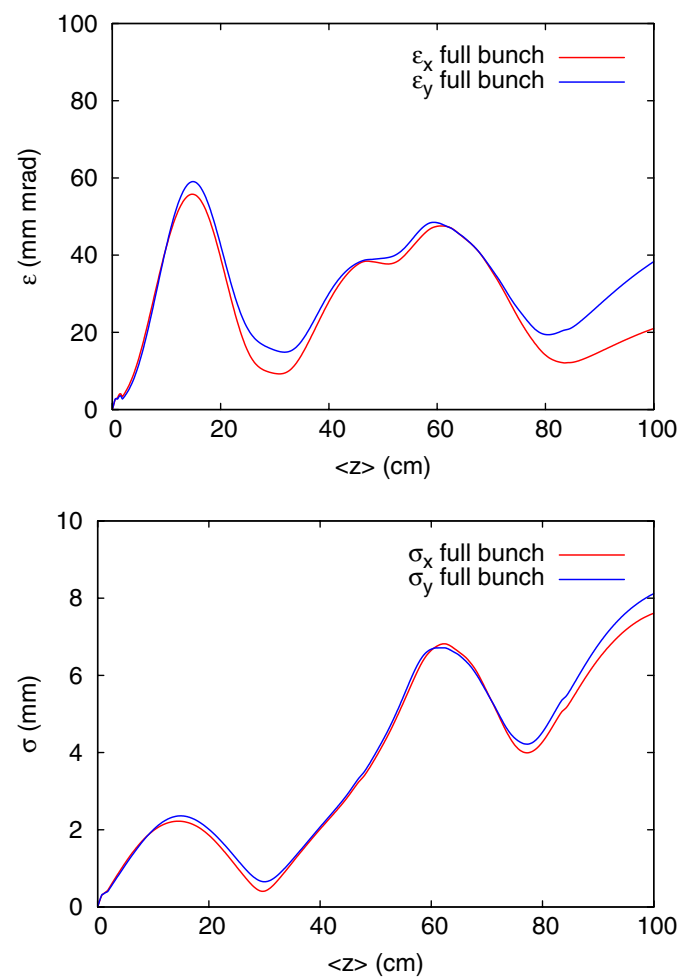

FIG. 6. Upper frame: Plot of the transverse emittances $\epsilon_{x}, \epsilon_{y}$ in $\mathrm{mm} \mathrm{mrad}$ as a function of $\langle z\rangle$ in $\mathrm{cm}$ for an energy selection of the beam $3<E<60 \mathrm{MeV}$. Lower frame: Plot of the rms beam sizes $\sigma_{x}, \sigma_{y}$ in $\mathrm{mm}$ for the same energy selection. 

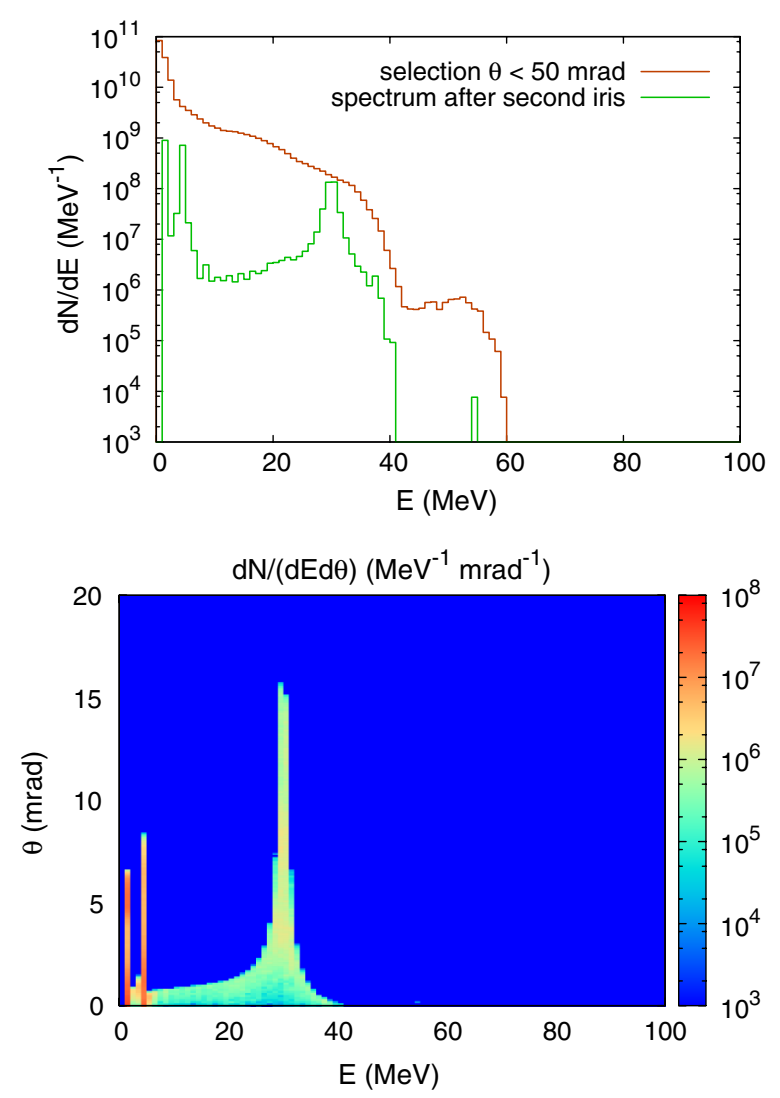

FIG. 7. Upper frame: Plot in a logarithmic scale of the proton energy spectrum after the second collimator (dark green curve) for the composite target foam + foil (TNSA) compared with the spectrum after the first collimator which selects the angle to $\theta<$ $50 \mathrm{mrad}$ (light brown curve). Lower frame: Plot of the energyangle distribution of the bunch after the second collimator in a logarithmic color scale.

the slow protons are still in the solenoid long after the center of mass of the bunch has overcome it. As a consequence, the interpretation of these plots is not intuitive. Exhaustive information can only be obtained from analyzing a full sequence of monochromatic energy slices.

The second collimator performs an energy selection as can be seen in Fig. 7, where we plot the energy distribution and the energy-angle distribution for the composite target (TNSA). In Fig. 8 the same distributions are shown for the target with critical density (MVA).

These figures show that the selection is successfully performed at $30 \mathrm{MeV}$ where a sharp peak is present, but the filter is not active at very low energies. Indeed at 2 and at $4 \mathrm{MeV}$ the focus of the solenoid is the same as for the $30 \mathrm{MeV}$ particles. These energies belong to a discrete spectrum with an accumulation point at zero energy. This can be easily checked analytically, working with a simplified model of a solenoid with sharp edges; see Appendix B. The presence of a low energy component is not desirable for the injection into a rf but it does not affect significantly the acceleration process as we will show later. The elimination
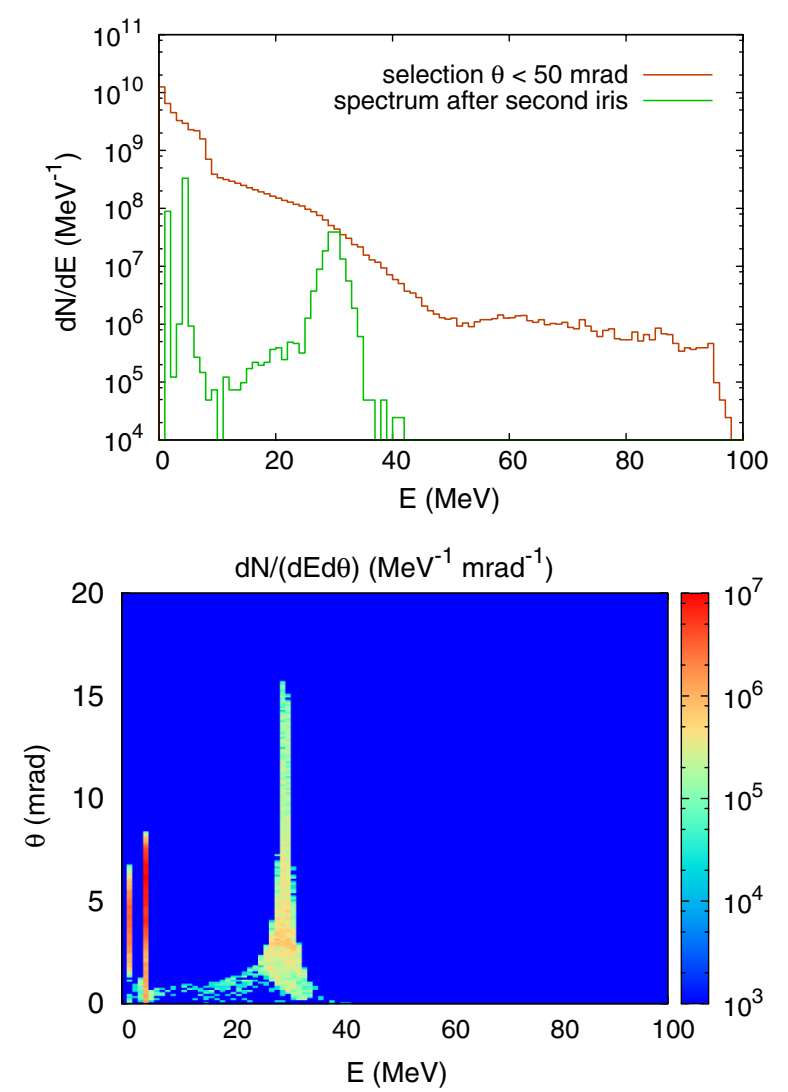

FIG. 8. Upper frame: Plot in a logarithmic scale of the proton energy spectrum after the second collimator (dark green curve) for the target with critical density (MVA) compared with the spectrum after the first collimator which selects the angle to $\theta<50 \mathrm{mrad}$ (light brown curve). Lower frame: Plot of the energy-angle distribution of the bunch after the second collimator in a logarithmic color scale.

of this component can be achieved with a weak chicane or a thin foil. The absorption of these components in the linac would not be a problem due to their low intensity.

We have also considered a similar transport scheme with two doublets of permanent magnetic quadrupoles of length $6 \mathrm{~cm}$ and gradients ranging from 80 to 120 Tesla/m such that the $x$ and $y$ focus is the same for $30 \mathrm{MeV}$ and comparable to the focus of the solenoid. Permanent magnetic quadrupoles are more stable and easier to handle. Their effectiveness in energy selection and focusing is however lower than the solenoid in all the configurations we have tried. An advantage is that all the low energy protons are filtered.

\section{POSTACCELERATION}

Starting from the bunch selected in energy by the previously described transport line, we have simulated the postacceleration in the linac ACLIP. This was conceived as a side coupled linac designed as a booster for a $30 \mathrm{MeV}$ proton injector working at $3 \mathrm{GHz}$. This high frequency 

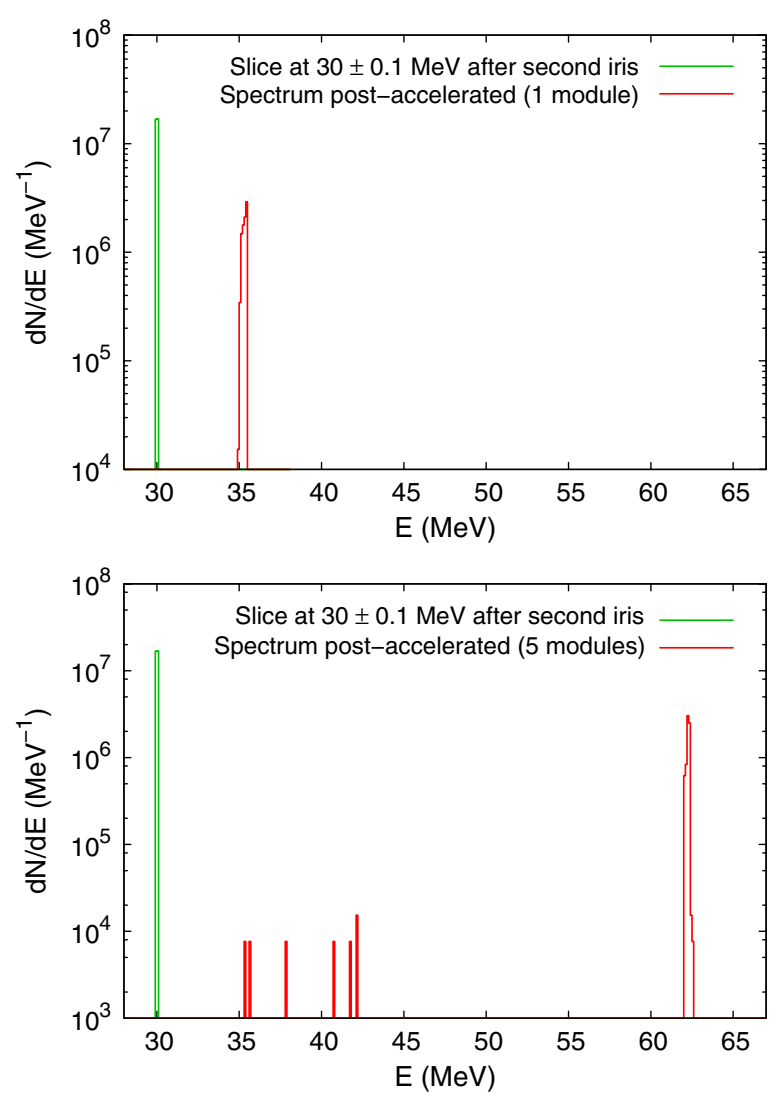

FIG. 9. Upper frame: Energy spectra for a bunch with initial energy of $30 \mathrm{MeV} \pm 0.1 \mathrm{MeV}$ (green line) postaccelerated in one module of ACLIP (red line). Lower frame: Postacceleration in five modules. The values refer to the actual number of particles for each bin and each bin has a width of $\Delta E=0.1 \mathrm{MeV}$.

implies a linac more compact and shorter than the standard lower-frequency proton linacs, used as injector of most synchrotrons, since the permissible accelerating field is roughly proportional to $f^{1 / 2}$ ( $f$ is the rf frequency) [44]. The final energy is $62 \mathrm{MeV}$. The linac consists of five different modules, each with a maximum of 30 accelerating cells arranged in two tanks, powered by a single rf feed. Beam focusing is obtained through the use of permanent magnetic quadrupoles (PMQs). The total length of the five modules is $3.1 \mathrm{~m}$. Eleven PMQs (gradient of $190 \mathrm{~T} / \mathrm{m}$ ) are positioned between the adjacent tanks and at the beginning
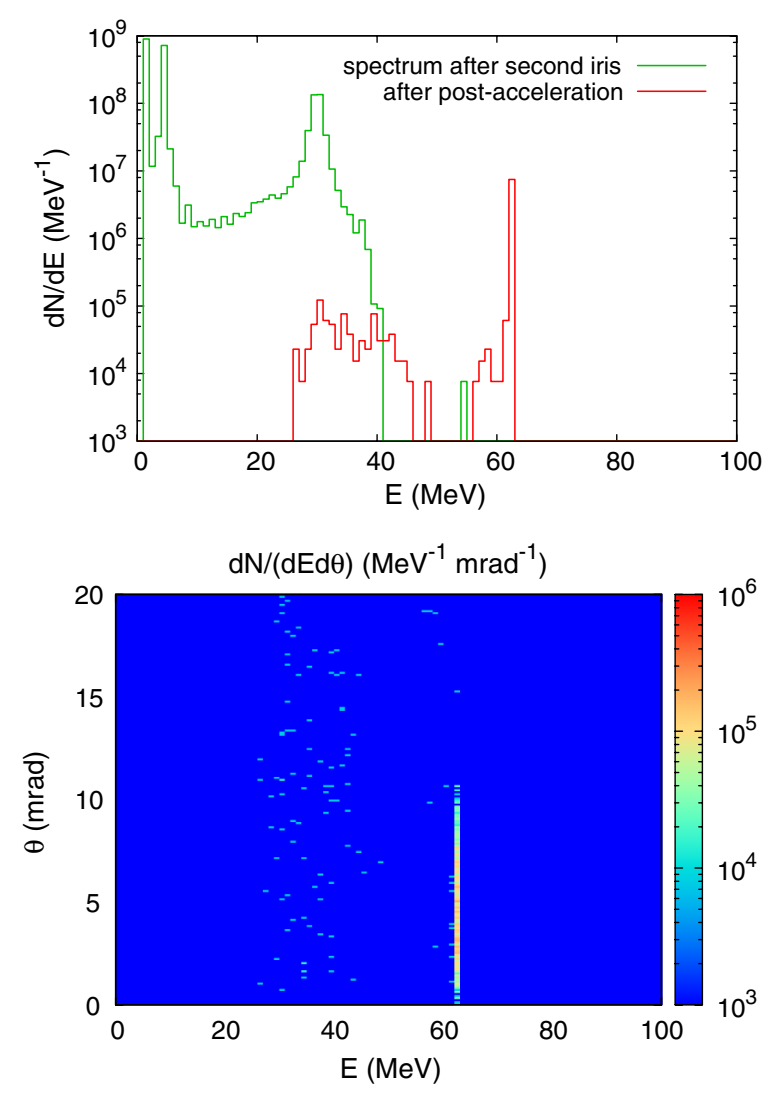

FIG. 10. Upper frame: Plot in a logarithmic scale of the proton energy spectrum after postacceleration by five modules of ACLIP (red curve) for the composite target foam + foil (TNSA) compared with the spectrum after the second collimator (dark green curve). Lower frame: Plot of the energy-angle distribution after postacceleration in a logarithmic color scale.

and the exit of ACLIP. All the modules are essentially identical, except for their progressive increase in length, due both to the increasing velocity of the protons and to the different numbers of accelerating cells. The rf design is based on the same mean accelerating field on axis in all ten tanks. The design foresees a peak surface field such that the bravery factor is 1.8 , with a mean axial field value of $E=20 \mathrm{MV} / \mathrm{m}$. The accelerating structure of each module consists of three basic elements: the basic cell plate, the bridge coupler, and the end cells.

TABLE I. Bunch parameters for the composite target (TNSA) at different stages. The values quoted in the columns for $N_{p \text { tot }}$ and $E_{p \text { tot }}$ refer to the whole proton bunch, with $E>1 \mathrm{MeV}$ for $z>0$. The values quoted in the columns $\epsilon_{x}, \epsilon_{y}, \Delta E / E, N_{p \text { (slice) }}$ refer to the energy slice defined in the last column. The laser energy is $6.75 \mathrm{~J}$. The energy spread $\Delta E$ for a distribution peaked at $E$ is defined by $d N / d E(E \pm \Delta E)=e^{-1} d N / d E(E)$.

\begin{tabular}{|c|c|c|c|c|c|c|c|}
\hline$\langle z\rangle(\mathrm{cm})$ & $N_{p \text { tot }}$ & $E_{p \text { tot }}(\mathrm{mJ})$ & $\epsilon_{x}(\mathrm{~mm} \mathrm{mrad})$ & $\epsilon_{y}(\mathrm{~mm} \mathrm{mrad})$ & $\Delta E / E$ & $N_{p(\text { slice })}$ & \\
\hline 0 & $3.5 \times 10^{11}$ & 195 & 0.021 & 0.023 & & $5.98 \times 10^{8}$ & at $30 \pm 1 \mathrm{MeV}$ \\
\hline 1.5 & $8.7 \times 10^{10}$ & 79 & 0.24 & 0.21 & & $3.58 \times 10^{8}$ & at $30 \pm 1 \mathrm{MeV}$ \\
\hline 84 & $2.1 \times 10^{9}$ & 2.8 & 0.48 & 1.91 & $3 \%$ & $2.69 \times 10^{8}$ & at $30 \pm 1 \mathrm{MeV}$ \\
\hline 400 & $8.4 \times 10^{6}$ & 0.08 & 0.71 & 0.39 & $0.2 \%$ & $7.53 \times 10^{6}$ & at $61 \pm 1 \mathrm{MeV}$ \\
\hline
\end{tabular}


The synchronization process between the laser pulse and the rf power amplifier is a major issue in this proposal. The phase acceptance window of the linac is of the order of $30 \mathrm{ps}$ and it turns out that the synchronization among laser pulses and rf fields has to be in the ps scale. Nevertheless, the Sorgente Pulsata Auto-amplificata di Radiazione Coerente (SPARC) Lab layout in Frascati already foresees devoted electronic and optical equipment to provide timing and synchronization in the femtosecond scale between $3 \mathrm{GHz}$ accelerating structures of the electron linac SPARC and the laser pulses. The fast timing structure has been developed to allow Thompson emission and plasma acceleration experiments, and we will use the same infrastructure, with minor changes, for our tests [45].

One of the critical issues of the hybrid acceleration scheme is compactness. The principal laser components can be located in a $20 \mathrm{~m}^{2}$ room. The interaction chamber, the transport line, and the linac (3.2 $\mathrm{m}$ long and less than $1 \mathrm{~m}$ wide) can be located in a radiation shielded room of a similar size. In our line the first module of the linac is located $2 \mathrm{~mm}$ after the second collimator.
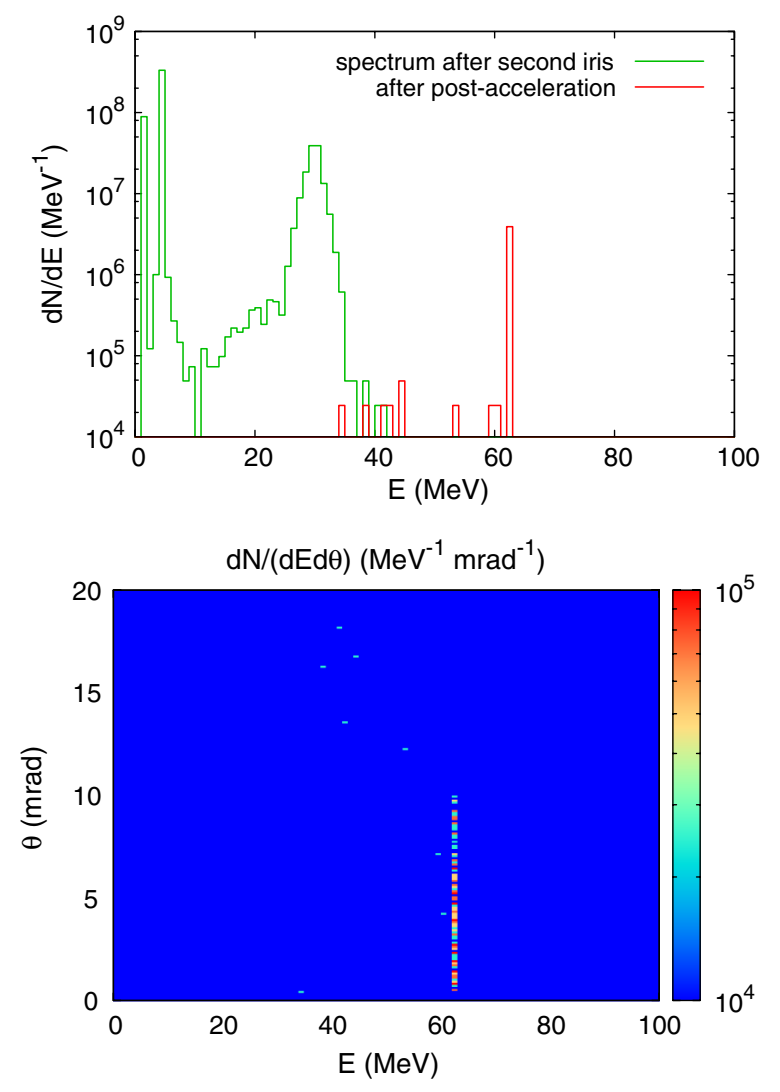

FIG. 11. Upper frame: Plot in a logarithmic scale of the proton energy spectrum after postacceleration by five modules of ACLIP (red curve) for the target with quasicritical density (MVA) compared with the spectrum after the second collimator (dark green curve). Lower frame: Plot of the energy-angle distribution after postacceleration in a logarithmic color scale.
The simulations of the postacceleration stages have been carried out using ASTRA [46]. In Fig. 9 (upper frame) we can see that if we inject a monochromatic bunch at $E=30 \mathrm{MeV}$ with a spread $\Delta E=0.1 \mathrm{MeV}$ into the first module of ACLIP, in phase with the field in the cavity, a good portion of it is accelerated up to $35 \mathrm{MeV}$. After five modules the bunch is accelerated up to $62 \mathrm{MeV}$ without additional losses; see Fig. 9 (lower frame).

The monochromatic bunch is just a slice of the whole bunch that propagates along our beam line. We have injected the whole bunch, obtained from the laser interaction with a composite foam + foil target (TNSA), coming out from the second collimator, and we found that a similar energy gain can be obtained. The final spectrum after five modules has a divergence less that $10 \mathrm{mrad}$; see Fig. 10 (lower frame). The energy distribution is peaked at $62 \mathrm{MeV}$ with a spread much less than $1 \mathrm{MeV}$ and has a low background; see Fig. 10 (upper frame). The number of protons in the peak is $\sim 10^{7}$. The main bunch parameters, including efficiency and energy spread, for the composite target (TNSA) at various stages (initial, after first collimator, second collimator, and at the end of the linac) are shown in Table I.

In Fig. 11 we consider the angle and energy distribution of the bunch obtained from the laser interaction with a target having critical density (MVA) postaccelerated after selection by the second collimator. We still observe the angle cutoff at $10 \mathrm{mrad}$, the energy peak at $62 \mathrm{MeV}$, and a negligible background. The total number of protons is comparable.

\section{CONCLUSIONS}

We have proposed a model for the transport of a laser accelerated beam based on a simple line consisting of a solenoid and two collimators. Starting from a laser pulse with $a=30$ and a composite target, we have shown that the average proton energy (temperature) can reach 7.2 $\mathrm{MeV}$ so that in a small slice around $30 \mathrm{MeV}$, with $\Delta E=1 \mathrm{MeV}$, the number of protons is $\sim 10^{8}$, after a collimation that cuts all the particles with a divergence above $50 \mathrm{mrad}$. The use of a second collimator on the focus of the solenoid for protons of $30 \mathrm{MeV}$ allows to select a bunch sharply peaked at this energy. The final beam has been injected into the linac and accelerated up to $35 \mathrm{MeV}$, with a single module, and up to $62 \mathrm{MeV}$, with five modules, without additional significant losses. The conclusion is that one can postaccelerate $\sim 10^{7}$ protons up to $62 \mathrm{MeV}$ with a small spread $\Delta E / E \ll 1 \%$ after an energy selection based on a solenoidal lens. These figures suggest that if the system works at $10 \mathrm{~Hz}$ for a couple of minutes the number of $62 \mathrm{MeV}$ protons delivered is $10^{10}$ corresponding to a total energy of $100 \mathrm{~mJ}$. The energy and the dose are at the threshold of clinical relevance. Acceleration to higher energies is possible using another stage such as the linac booster [47-49]. Supposing that these results can be 
confirmed in a single shot experiment, important technological developments are necessary to reach the $10 \mathrm{~Hz}$ maximum laser repetition rate in a stable and reliable way.

The start-to-end simulation was carried out in order to avoid initial guesses on the laser accelerated proton beam and to follow consistently the evolution along the transport line and the accelerating modules. The neglected space charge effects are not expected to be relevant. What might be more relevant is the structure of the targets we used. The density of the foil was chosen to be $n=40 n_{c}$ because the optimal thickness is not too low and the number of accelerated protons in the energy slice of interest is sufficiently high. Such targets are not presently available. Taking into account also recent experimental results [4], one may estimate that using presently available targets the number of protons after energy selection would be $\sim 10^{7}$ and decrease to $10^{6}$ or below after postacceleration. The composite targets we have considered are much less sensitive to the thickness and electron density of the foil. As a consequence, the results that we have obtained should be close to reality provided that a homogeneous foam layer can be prepared. The near critical targets, that are expected to be available soon from upgraded gas jets, have been modeled in a realistic way and might be the best candidates to provide a postaccelerated beam with $10^{7}$ protons at a high repetition rate.

\section{ACKNOWLEDGMENTS}

We would like to thank Professor P. Bolton (JAEA), Dr. A. Bacci (INFN), and Professor G. Dattoli (ENEA) for the continuous and profitable discussions. We acknowledge INFN for a grant supporting the LILIA experiment and CINECA (Centro Interuniversitario del Nord Est per il Calcolo Avanzato) Computing Center for the grant ISCRA Class A (Application Code HP10A0FQ14, Project Name PICPBA). We would like to thank the Italian Ministry of Foreign Affairs (MAE) for Grant No. PGR00079 20102012 Japan-PROMETHEUS: Research Laboratory for proton, ion and coherent $X$ radiation, based on laserplasma interaction. Laser injector formation requirements for post-acceleration supporting the scientific cooperation with Japan. This work has been partially supported by a grant of the Ministry of Education, University and Research-PRIN2009 (National Coordinator: S. Atzeni; Local Coordinator: G. Turchetti), under the title Study of shock induced inertial fusion and diagnostics based on proton bunches produced by laser beams.

\section{APPENDIX A: ENERGY SPECTRUM AND LONGITUDINAL DISTRIBUTION}

The basic one-dimensional model for TNSA is given by the Poisson-Boltzmann equation for the electrons heated by the laser pulse. Letting $V(z)$ be the electrostatic potential and imposing it vanishes jointly with its first derivative at some distance $z=h$ from the illuminated plane $z=0$, the solution of the Poisson-Boltzmann equation is determined and the proton energy is the potential difference between $z=0$ and $z=h$. As a consequence, according to [28] we have $E_{\max }=e V(0)=T \log \left(1+\tan ^{2} \alpha\right)$, where $T$ is the electron temperature and $\alpha=h /\left(\lambda_{D} \sqrt{2}\right)$, having denoted with $\lambda_{D}$ the Debye length. Choosing for instance $h=2 \lambda_{D}$ and noticing that $T \simeq m_{e} c^{2} a$, for $a \gg 1$ we have

$$
E_{\max }(\mathrm{MeV})=\frac{E_{\max }}{2 m_{e} c^{2}}=\frac{a}{2} \log \left(1+\tan ^{2} \sqrt{2}\right) \sim 2 a .
$$

The energy spectrum is approximated by $d N / d E=$ $C e^{-E / T}$, in which $C=\frac{N_{0}}{E_{0}}\left(e^{-E_{\min } / E_{0}}-e^{-E_{\max } / E_{0}}\right)^{-1}$, where $E_{0}$ is the mean energy and $N_{0}$ the total number of protons, if $E_{\min }<E<E_{\max }$ and vanishes elsewhere [28,29]. These results are still qualitatively correct when we consider a realistic three-dimensional case. An exponential spectrum with a high energy cutoff is still observed. The low energy cutoff appears only in special cases (mass limited targets). As a consequence, the energy spectrum is $\frac{d N}{d E}=C e^{-E / E_{0}}$ for $0<E<E_{\max }$ vanishing for $E>E_{\max }$. The average energy $\langle E\rangle \simeq E_{0}$ according to the one-dimensional model is given by $E_{0}=T=m_{e} c^{2} a$ so that $E_{0}(\mathrm{MeV})=$ $E_{0} /\left(2 m_{e} c^{2}\right)=a / 2$ and consequently $E_{0} / E_{\max }=1 / 4$. The energy spectra obtained from simulations and experiments are linear in $a$, to a good approximation, in a logarithmic scale and $E_{0}$ is determined by a fitting procedure. In both cases it is found that $E_{0} / E_{\max }$ varies between $1 / 8$ and 1/7; see for instance Table 1 in [3] and results described in [27] for more experimental evidences. The maximum energy $E_{\max }$ is still proportional to $a$ with a proportionality factor 2 for optimal targets so that in this case $E_{0} \simeq a / 4$. So, even though the scaling is the same as the one-dimensional model, the numerical factor is different. Since $E_{\max } \simeq 8 E_{0}$, a small error is made neglecting the cutoff and the distribution can be written

$$
\frac{d N}{d E}=\frac{N_{0}}{E_{0}} e^{-E / E_{0}},
$$

where $N_{0}$ is the total number of particles and $E_{0}$ is the average energy, which is the crucial parameter. Denoting with $\rho(E)$ the spectrum normalized to 1 , we have

$$
\rho(E)=\frac{1}{E_{0}} e^{-E / E_{0}} \quad\langle E\rangle=\int_{0}^{\infty} E \rho(E) d E=E_{0} .
$$

In order to compute the mean value of $z$ we recall that $\langle z\rangle=t\langle v\rangle$, where $v=(2 E / m)^{1 / 2}$. As a consequence, the longitudinal distribution for a bunch is given by

$$
\rho_{z}(z)=\int_{0}^{\infty} \delta(z-v t) \rho(E) d E .
$$

The mean value of $z$ is given by

$$
\langle z\rangle=\int_{-\infty}^{+\infty} z \rho(z) d z=t \int_{0}^{\infty} v \rho(E) d E=t\langle v\rangle .
$$


In a similar way we have

$$
\sigma_{z}^{2}=\left\langle z^{2}\right\rangle-\langle z\rangle^{2}=t^{2}\left(\left\langle v^{2}\right\rangle-\langle v\rangle^{2}\right), \quad \sigma_{z}=t \sigma_{v} .
$$

An easy computation gives $\langle v\rangle=v_{0} \sqrt{\pi} / 2$ and $\sigma_{v}=v_{0}(1-\pi / 4)^{1 / 2}$. Since $\sigma_{z} \sim\langle z\rangle / 2$ it is clear that the bunch becomes rapidly so long that the reference to $z$ in analyzing the transport does not elucidate the dynamics. If we cut the spectrum, as we have shown we can do with a solenoid, or at least break the bunch into quasimonochromatic slices, then their longitudinal extension is limited and they can be separately analyzed in a conventional way. We consider a slice of the bunch in $\left[E_{1}-\Delta E, E_{1}+\Delta E\right]$ so that the spectrum becomes

$$
\rho_{1}(E)=\frac{E_{0}^{-1}}{2 \operatorname{sh}\left(\delta_{0}\right)} e^{-\left(E-E_{1}\right) / E_{0}}, \quad \delta_{0}=\frac{\Delta E}{E_{0}} .
$$

The mean values of $v$ and $v^{2}$ are easily computed setting $v_{1}=\left(2 E_{1} / m\right)^{1 / 2}$. Changing the integration variable to $w=\left(E_{1}-E\right) / E_{0}$ so that $v=v_{1}\left(1-w E_{0} / E_{1}\right)^{1 / 2}$, we have

$$
\begin{aligned}
\langle v\rangle & =\frac{v_{1}}{2 \operatorname{sh}\left(\delta_{0}\right)} \int_{-\delta_{0}}^{\delta_{0}}\left(1-w \frac{E_{0}}{E_{1}}\right)^{1 / 2} e^{w} d w \\
& =v_{1}\left[1-\frac{\delta_{0}^{2}}{6}\left(\frac{E_{0}}{E_{1}}+\frac{1}{4} \frac{E_{0}^{2}}{E_{1}^{2}}\right)\right]
\end{aligned}
$$

We have expanded the integrand up to second order in $w$ and the result up to second order in $\delta_{0}$. In a similar way we have

$$
\left\langle v^{2}\right\rangle=\frac{v_{1}^{2}}{2 \operatorname{sh}\left(\delta_{0}\right)} \int_{-\delta_{0}}^{\delta_{0}}\left(1-w \frac{E_{0}}{E_{1}}\right) e^{w} d w=v_{1}^{2}\left(1-\frac{E_{0}}{E_{1}} \frac{\delta_{0}^{2}}{3}\right) .
$$

As a consequence, letting $\delta_{1}=\Delta E / E_{1}$, we finally obtain

$$
\left\langle v^{2}\right\rangle-\langle v\rangle^{2}=\frac{v_{1}^{2}}{12} \delta_{1}^{2}
$$

The final result reads

$$
\langle z\rangle=v_{1} t\left[1-\frac{\delta_{1}^{2}}{6}\left(\frac{1}{4}+\frac{E_{1}}{E_{0}}\right)\right], \quad \sigma_{z}=v_{1} t \frac{\delta_{1}}{\sqrt{12}} .
$$

We summarize the result writing $\langle z\rangle=v_{1} t+O\left(\delta_{1}^{2}\right)$ and $\sigma_{z}=\langle z\rangle \delta_{1} / \sqrt{12}$

\section{APPENDIX B: AN ANALYTICAL MODEL}

In the sharp edge approximation we can justify the presence of several peaks in the spectrum. For a given energy $E_{*}=30 \mathrm{MeV}$ we consider the focus of the solenoid which is located at $z_{\text {foc }}=D+L+D_{1}$, where $D_{1}$ is such that, from Eq. (3), we obtain $A\left(E_{*}\right)=0$ :

$$
z_{\mathrm{foc}}=D+L+\frac{D \cos \alpha+k^{-1 / 2} \sin \alpha}{D k^{1 / 2} \sin \alpha-\cos \alpha} .
$$

At $z=z_{\text {foc }}$ we place a collimator of radius $r$. A particle with energy $E \neq E_{*}$ and a given value of $\theta_{0}=\left(x_{0}^{\prime 2}+y_{0}^{\prime 2}\right)^{1 / 2}$ reaches the focal plane at a distance $d$ from the $z$ axis, where

$$
d=\left[x^{2}\left(z_{\mathrm{foc}}\right)+y^{2}\left(z_{\mathrm{foc}}\right)\right]^{1 / 2}=\theta_{0}|A(E)|
$$

and will go through the collimator only if $d<r$. As consequence the condition that the particle passes through the collimator is $d<r$.

We first consider the position of the focus $z_{\text {foc }}=$ $D+L+D_{1}$ as a function of $E$ and look for values of $E$ such that $z_{\text {foc }}(E)=z_{\text {foc }}\left(E_{*}\right)$. Indeed there are multiple values of the energy for which the same focus occurs. Choosing $D=2 \mathrm{~cm}, B_{0}=10$ Tesla, and $L=27 \mathrm{~cm}$, we find that at $E=30 \mathrm{MeV}$ with $x_{0}^{\prime}=y_{0}^{\prime}=0.05$, which corresponds to $p_{z}=0.2523$, we have $D_{1}=58.2 \mathrm{~cm}$ so that $z_{\text {foc }}=87.2 \mathrm{~cm}$. These values are somewhat different from the case with a smooth fringe field. Changing $x_{0}^{\prime}, y_{0}^{\prime}$ we change $p_{z}$ and the focus slightly changes. For instance, with $x_{0}^{\prime}=y_{0}^{\prime}=0.01$ we have $p_{z}=0.2528$ and $z_{\text {foc }}=$ $88.4 \mathrm{~cm}$. In Fig. 12 we show the position of the focus as a function of energy $E$ by keeping $x_{0}^{\prime}=y_{0}^{\prime}=0.01$. The same focus $z=88.4 \mathrm{~cm}$ occurring for $E=30 \mathrm{MeV}$ is also obtained for $E=4.3 \mathrm{MeV}$ and $E=1.6 \mathrm{MeV}$. The same figure shows the distance $d$ from the $z$ axis at
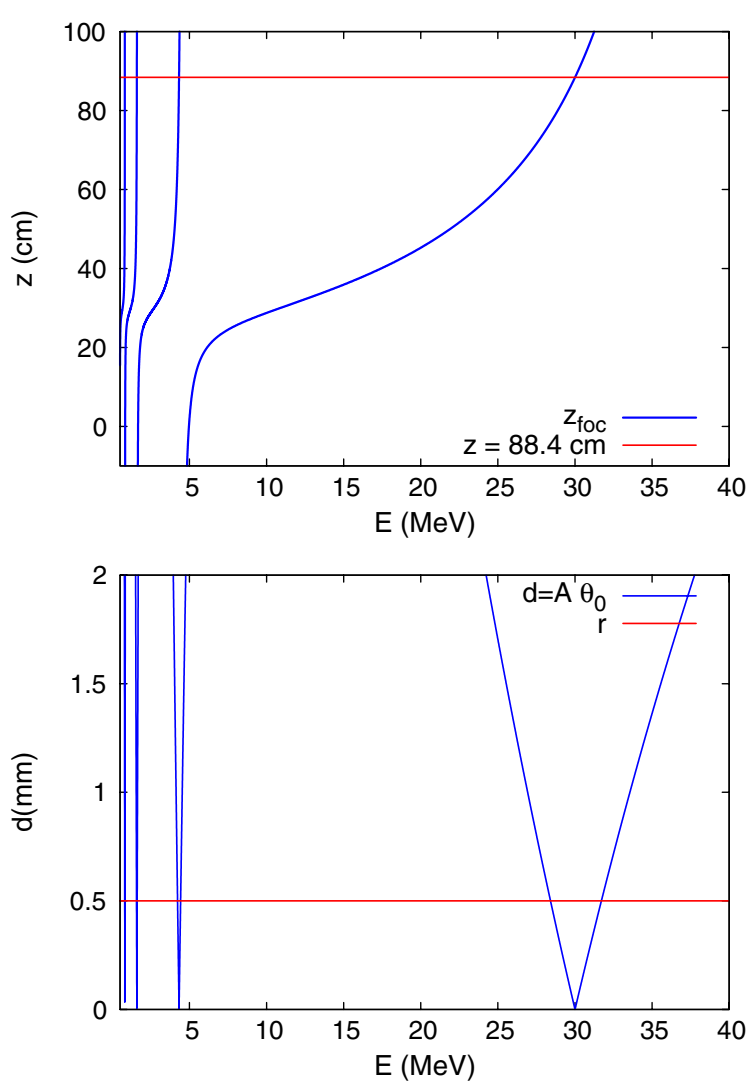

FIG. 12. Upper frame: Plot of the focus as a function of the energy compared with the focus for $E=30 \mathrm{MeV}$. Lower frame: comparison of the distance $d=A \theta_{0}$ from the $z$ axis at the point $z=88.4$ with the collimator radius $r=0.5 \mathrm{~mm}$ when the energy varies and $x_{0}^{\prime}=y_{0}^{\prime}=0.01$. 

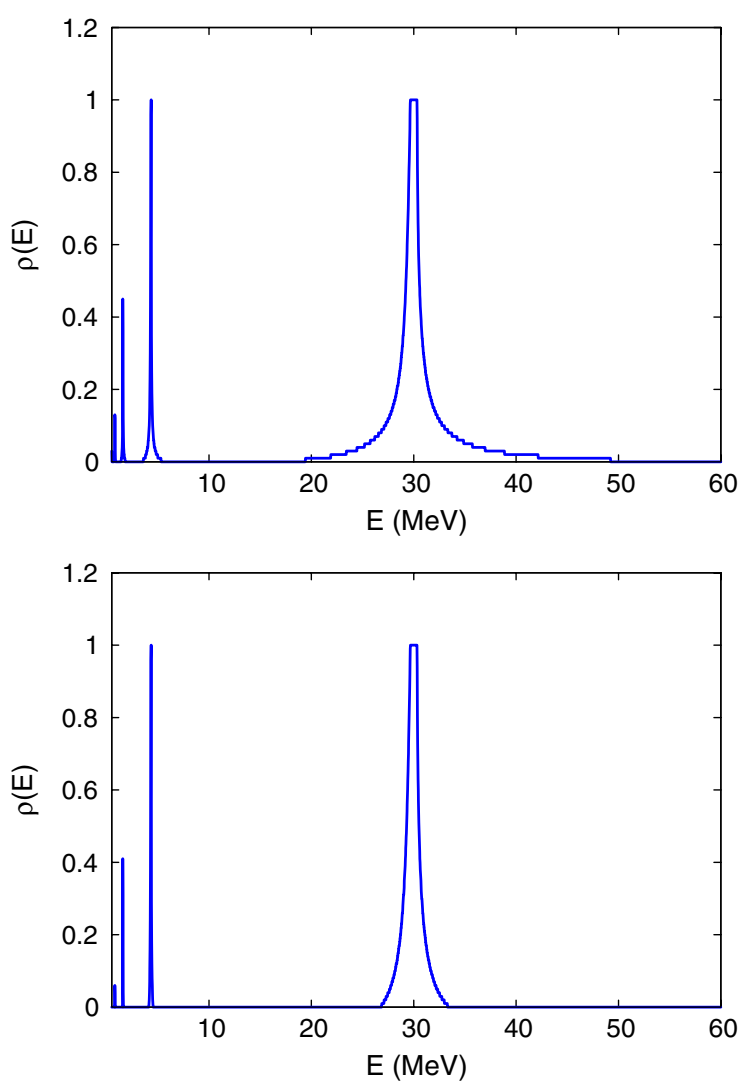

FIG. 13. Upper frame: Plot of the function $g(E)=\rho_{1}(E) /$ $\rho_{E}(E)$ which gives the fraction of particles having overcome the second collimator, at a given energy, whatever their initial value of angular deviation $\theta_{0}$. The spectrum is obtained for an angular distribution whose lower bound is $\theta_{0 \text { min }}=0.001$. Lower frame: The same as the upper frame with a larger value of the angular spectrum lower bound $\theta_{0 \min }=0.005$. In this case the spectrum is narrower.

$z=88.4 \mathrm{~cm}$ when the energy is varied and compares it with the collimator radius $r=0.5 \mathrm{~mm}$.

We consider now a beam having an exponential spectrum and a uniform angle distribution within a given range:

$$
\frac{d N}{d E d \theta_{0}}=N_{0} \rho\left(E, \theta_{0}\right) \int_{E_{\min }}^{E_{\max }} d E \int_{\theta_{0 \min }}^{\theta_{0 \max }} d \theta_{0} \rho\left(E, \theta_{0}\right)=1,
$$

where $N_{0}$ is the total number of particles having crossed the first collimator. We assume for simplicity a factorization of the distribution according to $\rho\left(E, \theta_{0}\right)=$ $\rho_{E}(E) \rho_{\theta}\left(\theta_{0}\right)$, where

$$
\rho_{E}(E)=\frac{1}{E_{0}} \frac{\exp \left(-E / E_{0}\right)}{e^{-E_{\min } / E_{0}-e^{-E_{\max } / E_{0}}}} \chi_{\left[E_{\min }, E_{\max }\right]}(E)
$$

and

$$
\rho_{\theta}\left(\theta_{0}\right)=\frac{1}{\theta_{0 \max }-\theta_{0 \min }} \chi_{\left[\theta_{0 \min }, \theta_{0 \max }\right]}\left(\theta_{0}\right)
$$

having defined $\chi_{[a, b]}(x)$ the characteristic function of the interval $[a, b]$. If $E_{\min } \ll E_{0}$ and $E_{\max } \gg E_{0}$, then
$e^{-E_{\min } / E_{0}}-e^{-E_{\max } / E_{0}} \simeq 1$. The spectrum at the exit of the second collimator at $z_{\mathrm{foc}}=L+D+D_{1}$ is given by

$$
\rho_{1}(E)=\int_{\theta_{0 \min }}^{\theta_{0 \max }} d \theta_{0} \vartheta\left(r-A \theta_{0}\right) \rho_{E}(E)=g(E) \rho_{E}(E) .
$$

The function $g(E)$ gives the fraction of particles transmitted at a given energy through the collimator. We recall that $A$ depends weakly on $\theta_{0}$ because $p_{z}=$ $p_{0}\left(1+\theta_{0}^{2}\right)^{-1 / 2}$ and for nonrelativistic particles $\beta_{z} \simeq p_{z}$ we have $k^{1 / 2}=\Omega_{L} / \beta_{z} \simeq\left(1+\theta_{0}^{2}\right)^{1 / 2} \Omega_{L} / p_{0}$ and $\theta_{0} \ll 1$. When $r$ decreases, the width of the peaks decreases too and a spectrum of lines is approached. When the value of $\theta_{0 \text { min }}$ decreases the peaks become larger and a background is progressively created because the particles almost parallel to the $z$ axis cross the collimators, whatever their energy. In Fig. 13 we show the function $g(E)$ for two different values of $\theta_{0 \text { min }}$ equal to 0.001 and 0.005 , respectively

[1] K. Zeil, S. D. Kraft, S. Bock, M. Bussmann, T. E. Cowan, T. Kluge, J. Metzkes, T. Richter, R. Sauerbrey, and U. Schramm, New J. Phys. 12, 045015 (2010).

[2] A. J. Mackinnon, Y. Sentoku, P. K. Patel, D. W. Price, S. Hatchett, M. H. Key, C. Andersen, R. Snavely, and R. R. Freeman, Phys. Rev. Lett. 88, 215006 (2002).

[3] M. Borghesi, J. Fuchs, S. V. Bulanov, A. J. Mackinnon, P. K. Patel, and M. Roth, Fusion Sci. Technol. 49, 412 (2006) [http://www.new.ans.org/pubs/journals/fst/a_1159].

[4] K. Ogura, M. Nishiuchi, A. S. Pirozhkov, T. Tanimoto, A. Sagisaka, T.Z. Esirkepov, M. Kando, T. Shizuma, T. Hayakawa, H. Kiriyama et al., Opt. Lett. 37, 2868 (2012).

[5] P. Antici, M. Fazi, A. Lombardi, M. Migliorati, L. Palumbo, P. Audebert, and J. Fuchs, IEEE Trans. Plasma Sci. 36, 1843 (2008).

[6] P. Antici, M. Migliorati, A. Mostacci, L. Picardi, L. Palumbo, and C. Ronsivalle, Phys. Plasmas 18, 073103 (2011).

[7] P. Londrillo, G. Servizi, A. Sgattoni, S. Sinigardi, M. Sumini, and G. Turchetti, $\mathrm{CO}_{2}$ Laser Optimization and Applications (Intech, Rijeka, Croatia, 2011), Chap. 17, ISBN 798-953-307-925.

[8] A. Bacci, D. Batani, G. P. Cirrone, C. De Martinis, D. Delle Side, A. Fazzi, D. Giove, D. Giulietti, L. Gizzi, L. Labate et al., in 2nd Workshop-Plasmi, Sorgenti, Biofisica ed Applicazioni, Lecce, Italy (ESE-University Publishing, Salento, 2012), pp. 136-143, ISBN: 978-888305-087-9.

[9] V. G. Vaccaro, M. R. Masullo, C. De Martinis, L. Gini, D. Giove, A. Rainò, V. Variale, L. Calabretta, A. Rovelli, S. Barone et al., in Proceedings of the 25th International Linear Accelerator Conference LINAC2010, Tsukuba, Japan (KEK, Tsukuba, Japan, 2010), pp. 467-469 [http://accelconf.web.cern.ch/AccelConf/LINAC2010/ papers/tup031.pdf].

[10] M. Nishiuchi, H. Sakaki, T. Hori, P. R. Bolton, K. Ogura, A. Sagisaka, A. Yogo, M. Mori, S. Orimo, A. S. Pirozhkov et al., Phys. Rev. ST Accel. Beams 13, 071304 (2010). 
[11] M. Ikegami, S. Nakamura, Y. Iwashita, T. Shirai, H. Souda, Y. Tajima, M. Tanabe, H. Tongu, H. Itoh, H. Shintaku et al., Phys. Rev. ST Accel. Beams 12, 063501 (2009).

[12] A. Noda, Y. Iwashita, H. Souda, H. Tongu, A. Wakita, H. Daido, M. Ikegami, H. Kiriyama, M. Mori, M. Nishiuchi et al., in Proceedings of LINAC08 Victoria, BC, Canada (2008), MOP060, p. 216.

[13] M. Schollmeier, S. Becker, M. Geißel, K. A. Flippo, A. Blažević, S. A. Gaillard, D. C. Gautier, F. Grüner, K. Harres, M. Kimmel et al., Phys. Rev. Lett. 101, 055004 (2008).

[14] M. Roth, I. Alber, V. Bagnoud, C. R. D. Brown, R. Clarke, H. Daido, J. Fernandez, K. Flippo, S. Gaillard, C. Gauthier et al., Plasma Phys. Controlled Fusion 51, 124039 (2009).

[15] K. Harres, I. Alber, A. Tauschwitz, V. Bagnoud, H. Daido, M. Gunther, F. Nurnberg, A. Otten, M. Schollmeier, J. Schutrumpf et al., Phys. Plasmas 17, 023107 (2010).

[16] T. Burris-Mog, K. Harres, F. Nürnberg, S. Busold, M. Bussmann, O. Deppert, G. Hoffmeister, M. Joost, M. Sobiella, A. Tauschwitz et al., Phys. Rev. ST Accel. Beams 14, 121301 (2011).

[17] T. Nakamura, S. V. Bulanov, T.Z. Esirkepov, and M. Kando, Phys. Rev. Lett. 105, 135002 (2010).

[18] A. Yogo, H. Daido, S. V. Bulanov, K. Nemoto, Y. Oishi, T. Nayuki, T. Fujii, K. Ogura, S. Orimo, A. Sagisaka et al., Phys. Rev. E 77, 016401 (2008).

[19] T. Esirkepov, M. Yamagiwa, and T. Tajima, Phys. Rev. Lett. 96, 105001 (2006).

[20] P. Londrillo, F. Rossi, G. Servizi, S. Sinigardi, G. Turchetti, and P. Bolton (unpublished).

[21] A. Henig, D. Kiefer, K. Markey, D. C. Gautier, K. A. Flippo, S. Letzring, R. P. Johnson, T. Shimada, L. Yin, B. J. Albright et al., Phys. Rev. Lett. 103, 045002 (2009).

[22] T. Ceccotti, A. Lévy, H. Popescu, F. Réau, P. D’Oliveira, P. Monot, J. P. Geindre, E. Lefebvre, and P. Martin, Phys. Rev. Lett. 99, 185002 (2007).

[23] T. Nakamura, M. Tampo, R. Kodama, S. V. Bulanov, and M. Kando, Phys. Plasmas 17, 113107 (2010).

[24] A. Sgattoni, P. Londrillo, A. Macchi, and M. Passoni, Phys. Rev. E 85, 036405 (2012).

[25] A. Brantov and V. Bychenkov, Plasma Phys. Rep. 36, 256 (2010).

[26] Y. Fukuda, A. Y. Faenov, M. Tampo, T. A. Pikuz, T. Nakamura, M. Kando, Y. Hayashi, A. Yogo, H. Sakaki, T. Kameshima et al., Phys. Rev. Lett. 103, 165002 (2009).

[27] H. Daido, M. Nishiuchi, and A. S. Pirozhkov, Rep. Prog. Phys. 75, 056401 (2012).

[28] M. Passoni and M. Lontano, Laser Part. Beams 22, 163 (2004).

[29] M. Passoni and M. Lontano, Phys. Rev. Lett. 101, 115001 (2008).

[30] A. Macchi and C. Benedetti, Nucl. Instrum. Methods Phys. Res., Sect. A 620, 41 (2010).

[31] B. Qiao, M. Zepf, M. Borghesi, and M. Geissler, Phys. Rev. Lett. 102, 145002 (2009).

[32] D. Jung, L. Yin, B. J. Albright, D. C. Gautier, R. Hörlein, D. Kiefer, A. Henig, R. Johnson, S. Letzring, S. Palaniyappan et al., Phys. Rev. Lett. 107, 115002 (2011).
[33] I. Hofmann, J. Meyer-ter Vehn, X. Yan, A. Orzhekhovskaya, and S. Yaramyshev, Phys. Rev. ST Accel. Beams 14, 031304 (2011).

[34] I. Hofmann, A. Orzhekhovskaya, S. Yaramyshev, M. Roth, and M. Droba, in Proceedings of HIAT09, Venice, Italy (2009) [http://accelconf.web.cern.ch/AccelConf/ HIAT2009/index.htm].

[35] I. Hofmann, J. M. ter Vehn, X. Yan, and H. Al-Omari, Nucl. Instrum. Methods Phys. Res., Sect. A 681, 44 (2012).

[36] V. G. Vaccaro, M. R. Masullo, C. De Martinis, D. Giove, A. Rainò, V. Variale, S. Mathot, R. J. Rush, and M. Iskander, in Proceedings of the 23rd Particle Accelerator Conference, Vancouver, Canada, 2009 (IEEE, Piscataway, NJ, 2009) [http://accelconf.web .cern.ch/AccelConf/PAC2009/papers/fr5rep075.pdf].

[37] V. G. Vaccaro, R. Buiano, A. D’Elia, G. De Michele, M. R. Masullo, F. Alessandria, D. Giove, C. De Martinis, E. Di Betta, M. Mauri et al., in Proceedings of 18th International Conference on Cyclotrons and their Applications, Catania, Italy, 2007, edited by D. Rifuggiato and L.A.C. Piazza (INFN-LNS, Catania, Italy, 2008), pp. 172-174 [http://accelconf.web.cern.ch/ AccelConf/c07/PAPERS/172.pdf].

[38] A. V. Brantov, V. Y. Bychenkov, and V. Rozmus, Quantum Electron. 37, 863 (2007).

[39] C. Benedetti, A. Sgattoni, G. Turchetti, and P. Londrillo, IEEE Trans. Plasma Sci. 36, 1790 (2008).

[40] C. Benedetti, P. Londrillo, L. Rossi, and G. Turchetti, Commun. Nonlinear Sci. Numer. Simul. 13, 204 (2008).

[41] C. Benedetti, P. Londrillo, L. Rossi, and G. Turchetti, Commun. Nonlinear Sci. Numer. Simul. 13, 209 (2008).

[42] P. Londrillo, C. Benedetti, A. Sgattoni, and G. Turchetti, Nucl. Instrum. Methods Phys. Res., Sect. A 620, 28 (2010).

[43] T. Toncian, M. Borghesi, J. Fuchs, E. d'Humires, P. Antici, P. Audebert, E. Brambrink, C. A. Cecchetti, A. Pipahl, L. Romagnani et al., Science 312, 410 (2006).

[44] W. D. Kilpatrick, Rev. Sci. Instrum. 28, 824 (1957).

[45] A. Gallo, D. Alesini, M. Bellaveglia, G. Gatti, and C. Vicario, in Proceedings of the 11th European Particle Accelerator Conference, Genoa, 2008 (EPS-AG, Genoa, Italy, 2008), pp. 3354-3356 [http://accelconf.web.cern.ch/ AccelConf/e08/papers/thpc156.pdf].

[46] K. Flöttmann, S. M. Lidia, and P. Piot, in Proceedings of the 20th Particle Accelerator Conference, Portland, OR, 2003 (IEEE, New York, 2003), pp. 3500-3502.

[47] C. De Martinis, C. Birattari, D. Giove, L. Serafini, P. Berra, E. Rosso, B. Szeless, U. Amaldi, K. Crandall, M. Mauri et al., in Proceedings of the 8th European Particle Accelerator Conference, Paris, 2002 (EPS-IGA and CERN, Geneva, 2002), pp. 2717-2729 [http://accelconf .web.cern.ch/AccelConf/e02/PAPERS/MOPRI095.pdf].

[48] U. Amaldi, P. Berra, K. Crandall, D. Toet, M. Weiss, R. Zennaro, E. Rosso, B. Szeless, M. Vretenar, C. Cicardi et al., Nucl. Instrum. Methods Phys. Res., Sect. A 521, 512 (2004).

[49] C. De Martinis, D. Giove, U. Amaldi, P. Berra, K. Crandall, M. Mauri, M. Weiss, R. Zennaro, E. Rosso, B. Szeless et al., Nucl. Instrum. Methods Phys. Res., Sect. A 681, 10 (2012). 